\section{La atención a la enfermedad renal en Uruguay: la perspectiva de individuos con trasplante renal}

\author{
Care for chronic kidney disease in Uruguay: \\ the perspective of kidney transplant patients
}

\author{
A atenção à insuficiência renal no Uruguai: \\ a perspectiva de indivíduos com \\ transplante renal
}

Francisco Javier Mercado-Martínez 1

Rodolfo Levin-Echeverri 2

doi: 10.1590/0102-311X00160416

\section{Resumen}

Este trabajo examina la atención renal desde la perspectiva de quienes han sido trasplantados, padeciendo enfermedad renal crónica (ERC), así como la de sus familias. Se realizó un estudio cualitativo en tres ciudades de Uruguay. Utilizando un muestreo teórico, seleccionamos 25 personas con ERC que vivieran con trasplante renal, y cinco familiares. Se hicieron entrevistas semiestructuradas para explorar las perspectivas de los participantes; así como análisis de contenido. Cuatro ejes temáticos fueron construidos a partir del discurso de los participantes: reconociendo los logros del sistema de atención, teniendo derecho a la salud, identificando problemas en la atención y formulando propuestas de acción. El sistema de atención es considerado adecuado, eficiente $y$ de calidad por quienes viven con trasplante renal, son jóvenes, trabajadores, de Montevideo y están cubiertos por el sector sanitario privado. Lo contrario sucede con los jubilados o pensionistas, con multi-morbilidad, del interior del país, cubiertos por el sector público y viviendo en la pobreza. Los participantes también formulan propuestas para resolver los problemas identificados. Además de reconocer el acceso gratuito y universal a las terapias renales, a las cuales consideran tener derecho, concluimos que los individuos con trasplante de riñón valoran favorablemente la atención sanitaria y los organismos involucrados. Sin embargo, algunos enfrentan serios obstáculos para acceder a una atención gratuita, oportuna y de calidad. Recomendamos impulsar estrategias para incorporar propuestas de los individuos enfermos $y$ sus familias, a fin de mejorar dicha atención y disminuir las desigualdades sociales.

Insuficiencia Renal Crónica, Transplante de Riñón, Sistemas de Salud

\author{
Correspondencia \\ F. J. Mercado-Martínez \\ Universidad de Guadalajara. \\ Mar Egeo 1452-41, Country Club, Guadalajara, Jalisco - \\ 44610, México. \\ fjaviermercado@yahoo.com.mx \\ 1 Universidad de Guadalajara, Guadalajara, México. \\ 2 Instituto de Higiene, Universidad de la República, Montevideo, \\ Uruguay.
}


La enfermedad renal crónica (ERC) se ha convertido en un problema de salud prioritario a nivel mundial; sus efectos se observan en los individuos que la padecen, sus familias, el sistema sanitario y la sociedad en su conjunto ${ }^{1}$. Las terapias renales sustitutivas (TRS) constituyen la alternativa disponible para su tratamiento; siendo el trasplante renal la mejor opción terapéutica según los profesionales de la salud 2. Sin embargo, el mismo se ve limitado por múltiples factores, destacando la poca disponibilidad de órganos, sus costos elevados y la carencia de recursos económicos. Por ello, los individuos enfermos suelen enfrentar obstáculos para acceder al trasplante o seguir el tratamiento y evitar el rechazo del órgano implantado ${ }^{3}$.

Las TRS han sido objeto de creciente atención y mejoras, sobre todo en los países desarrollados. A la vez, han enfrentado múltiples desafíos en esos países, particularmente en aquellos en vías de desarrollo. Por ejemplo, se estima que dos tercios de los países de bajos ingresos no tienen acceso a tales terapias 4; su financiamiento, costo y cobertura difiere en países con un sistema de sanidad público respecto a aquellos con uno mixto o privado $5 \mathrm{y}$ las personas con ERC que viven en áreas remotas reciben menos atención de médicos especialistas, estudios de laboratorio y medicamentos, respecto a quienes habitan en centros urbanos 6 .

Aunado a lo anterior, pocos esfuerzos sistemáticos se han llevado a cabo para incorporar el punto de vista y las propuestas de la población sobre la atención sanitaria vinculada a tales terapias ${ }^{7}$. Tres posturas de los profesionales sanitarios destacan en relación a los enfermos con trasplante renal: algunos profesionales argumentan que los enfermos vuelven a la normalidad una vez trasplantados 8 ; otros sostienen que la no adherencia al tratamiento médico, y el consecuente rechazo de los órganos, se deben a la falta de interés y apatía de enfermos y familiares 9 ; otros más, apoyándose en encuestas de satisfacción, concluyen que quienes han sido trasplantados están más satisfechos con la atención médica que aquellos en diálisis 10. Sin embargo, diversos autores cuestionan los señalamientos anteriores y sus supuestos, argumentando la necesidad de replantear la forma de examinar el tema 11. Entre otras cosas, destacan la importancia de comprender el punto de vista, las experiencias y prácticas de los actores sociales involucrados en los procesos sanitarios, incluyendo a la población enferma. Ello debido a que generalmente se les deja de lado o minimiza, a pesar de llamados de organismos internacionales que consideran la participación social como elemento clave para la modernización y la reforma sanitaria 12. Por lo anterior, este trabajo tiene como objetivo examinar la atención renal desde la óptica de quienes padeciendo ERC han sido trasplantados, así como la de sus familias.

Decidimos realizar el estudio en Uruguay por tres motivos: por ser un país con un sistema sanitario con cobertura gratuita y universal 13; por ser líder en materia de donación y trasplante de riñón en América Latina 14, y porque la participación de los usuarios organizados aparece como un carácter distintivo de la reforma sanitaria implementada del 2006 en adelante 15.

\section{Notas sobre el sistema sanitario uruguayo}

El sistema de salud uruguayo se estructuró sobre tres pilares básicos durante el siglo XX: el sector mutual, el sector público y el sector de seguros privados. El primero se cimentó en las comunidades de inmigrantes y cooperativas médicas que conformaron un modelo relativamente exitoso hasta mediados de siglo. El segundo correspondió -en distintas versiones- a los servicios asistenciales orientados a trabajadores de la administración pública y a los grupos sociales más desfavorecidos. El tercero a los seguros privados, históricamente con una presencia mínima.

El Frente Amplio, fuerza política de izquierda, asume el gobierno en las elecciones de 2004 con un programa político en el cual la reforma sanitaria se convierte en una de sus principales iniciativas. Se fundamenta en que dicho sistema debe ser equitativo en la forma como las personas contribuyan al financiamiento de la atención y responder a las expectativas de la población. En dicho marco se entiende la salud como núcleo de las políticas sociales 16. A partir del 2007 se lleva a cabo la Reforma del Sistema de Salud, caracterizándose por integrar al sector público, las mutuales y los seguros privados en el denominado Sistema Nacional Integrado de Salud (SNIS) 17. Se trata de un sistema orientado a resolver la fragmentación de la atención médica, racionalizar los servicios, ahorrar costos, mejorar la equidad en el acceso a las prestaciones y hacerlo eficiente. 
Tal estructura supuso la creación de un fondo único de financiamiento (Fondo Nacional de Salud -FONASA), articulador de los sectores mencionados. En paralelo se llevó a cabo una reforma tributaria que conllevó un cambio significativo en el aporte a la Seguridad Social; ello en tanto que, a medida que aumenta el ingreso personal, se incrementa el aporte a la misma y por tanto el porcentaje destinado al FONASA.

La totalidad de la población tiene cobertura asistencial en cualquiera de los sectores. El ciudadano tiene la opción de elegir su afiliación a uno u otro; ya que a partir de la reforma desaparece cualquier posibilidad de selección adversa por parte de las instituciones de salud. En tal contexto, el sector público brinda asistencia aproximadamente a $40 \%$ de la población; se trata de aquellos con menos recursos y sin cobertura de la seguridad social. Está compuesto por varias instituciones, siendo la de mayor peso la denominada Administración de Seguros del Estado (ASSE) y a la que se le conoce popularmente como Salud Pública. Su financiamiento proviene de la asignación presupuestaria del Estado. En cambio, el sector mutual cubre aproximadamente a un 56\% de la población; básicamente a trabajadores formales y sus familias y a beneficiarios de la seguridad social. Está compuesto por las llamadas Instituciones de Asistencia Médica Colectiva (IAMCs) -conocidas como mutualistas o sociedades médicas-. Estas instituciones son entidades prestadoras de servicios de salud, que dependen mayoritariamente del FONASA al recibir un pago per cápita, pero el asegurado cubre una sobre cuota y un pago por los servicios -denominado ticket- y por los medicamentos. Finalmente, los seguros privados de cobertura total cubren aproximadamente al 3\% de la población; o sea, al sector con mayor capacidad económica.

A casi una década de haber sido implementada, la reforma sanitaria constituye un espacio permanente para la discusión académica y la decisión política 18 . En dicho contexto debe comprenderse la atención a los enfermos individuos con enfermedad renal y a las instituciones involucradas. El Fondo Nacional de Recursos (FNR), por ejemplo, ha sido actor clave en el sector sanitario, particularmente en el tratamiento renal. Se trata de una persona pública no estatal que brinda cobertura financiera a los procedimientos de medicina altamente especializada y a los medicamentos de alto costo de toda la población. Los procedimientos cubiertos se efectúan a través de los llamados Institutos de Medicina Altamente Especializada (IMAEs), pero el FNR los financia 19.

El Instituto Nacional de Donación y Trasplante de Células, Tejidos y Órganos (INDT) también ha jugado un papel central en la donación y trasplante de órganos en el país. Entre otras funciones, se encarga de promover y organizar la lista de donantes, así como realizar procedimientos de extracción, conservación y preparación, abastecimiento oportuno y adecuado de órganos, tejidos y células para su implante. Ambos organismos (FNR y INDT) se ubican en la capital, Montevideo.

Según la información disponible, a fines de 2015 había aproximadamente 3.000 personas con algún grado de enfermedad renal, de las cuales cerca de 1.200 estaban en terapia dialítica. Y entre 1969, año en que se hizo el primer trasplante de riñón en el país, y el 2015 se realizaron 2.034 trasplantes del mismo órgano 20 . Todos los gastos fueron cubiertos por el FNR.

\section{Materiales y métodos}

Bajo un enfoque crítico interpretativo 21, realizamos un estudio cualitativo, al considerarlos el marco teórico y la estrategia idóneas para explorar el punto de vista de los actores sociales sobre la atención renal. Utilizando un muestreo propositivo 22 , seleccionamos individuos con insuficiencia renal que habían sido trasplantados -y sus familiares-, tanto de Montevideo, la capital, como de Paysandú y Rivera, ciudades del interior, ubicadas a 374 y 650 kilómetros de la primera. Seleccionamos 30 personas -25 enfermos y cinco familiares. Once eran hombres y 19 mujeres; sus edades oscilaban entre 30 y 79 años; 13 vivían en Montevideo, 12 en Rivera y cinco en Paysandú; 13 estaban afiliados al sector público -ASSE- y 17 a las mutuales.

El trabajo de campo lo realizamos entre abril y julio de 2014. Empleando el formato de una entrevista semiestructurada, el primer autor invitó a los participantes a narrar su historia desde el inicio de su enfermedad. El tipo de preguntas formuladas fue: ¿Me podría contar como ha sido su vida desde que le diagnosticaron su enfermedad? ¿Cuáles han sido los principales problemas que ha tenido desde que fue trasplantado/a? ¿Podría explicarme con detalle en qué consiste esa dificultad? Para este trabajo 
sólo se utilizó información sobre la atención sanitaria. Cada participante decidió el lugar y la hora de la entrevista. Por esta razón se hicieron en sus casas, en la sede de ATUR (Asociación de Trasplantados del Uruguay) o en un lugar público. Con la excepción de dos participantes que estuvieron acompañados por un familiar, las entrevistas se hicieron individualmente. Su duración fue de dos horas en promedio.

Las entrevistas fueron audio-grabadas, luego transcritas por tres personas capacitadas. Se utilizó el programa Ethnograph 6.0 (Qualis Research; http://www.qualisresearch.com/default.htm) y se hizo análisis de contenido convencional 23. El procedimiento consistió en que el primer autor leyó repetidamente las entrevistas para lograr una inmersión en los datos y una visión de la información en su conjunto. Luego procedió a generar códigos provisionales con sus correspondientes etiquetas. Progresivamente fueron emergiendo los temas generales o ejes temáticos. A lo largo del proceso, los autores nos reunimos varias veces para dialogar sobre los procedimientos seguidos y los resultados obtenidos, lo cual retroalimentó el proceso. Una vez acordados los ejes temáticos y su contenido correspondiente, seleccionamos fragmentos de las entrevistas para utilizarlos como soporte empírico.

El proyecto fue evaluado y aprobado por dos comités de ética, uno de la Universidad de Guadalajara (México) y otro de la Universidad de la República (Uruguay). Una vez habiéndoles explicitado el proyecto, y entregado una síntesis del mismo por escrito, se invitó a las personas seleccionadas a participar y, una vez habiendo aceptado, a dar su permiso para grabar. Se les hizo énfasis en el anonimato y la confidencialidad de la información.

\section{Resultados}

Cuatro ejes temáticos fueron construidos a partir del discurso de los participantes sobre la atención sanitaria otorgada en torno al trasplante renal: reconociendo los logros del sistema de atención, teniendo derecho a la salud, enfrentando problemas en la atención y formulando propuestas de acción.

\section{Reconociendo los logros del sistema de atención (renal)}

El primer eje temático alude al reconocimiento de los participantes de los logros del sistema sanitario en la atención renal. Tal reconocimiento abarca cinco subtemas: la superioridad del sistema de salud uruguayo, el papel del FNR, la transparencia del INDT, el trato de los profesionales de la salud y los resultados exitosos de los trasplantes.

El sistema sanitario uruguayo, específicamente la atención brindada a quienes padecen insuficiencia renal, es valorado positivamente al considerarlo superior a los sistemas de países de la región, tales como los de Paraguay, Perú y Bolivia. El argumento dado es que dicho sistema ofrece acceso universal y gratuito a la atención médica, a diferencia de lo que ocurre en tales países. Cabe notar que no hacen comparación alguna con el sistema de salud argentino o el brasileño, aunque el primero aparece con frecuencia en sus historias, sea porque varios fueron allá en búsqueda de un diagnóstico complementario o estuvieron en tratamiento.

El FNR es objeto de reconocimiento al ser la instancia encargada de cubrir los costos de las terapias renales. Quienes se refieren al mismo señalan que, de no contarse con dicho fondo, las personas no tendrían los recursos para pagar la diálisis, ni el trasplante. Por tal motivo, quienes iniciaron la diálisis y/o fueron trasplantados antes de la reforma sanitaria del 2006 no encuentran diferencia alguna entre la situación que prevalecía antes y después de la misma, porque el FNR cubría los costos de las terapias renales desde principios de los 1980.

El INDT es objeto de reconocimiento por otro motivo, la confianza que les merece. Según las personas entrevistadas, este Instituto actúa con transparencia por la forma como funciona, y por no haber motivo alguno para cuestionarle o dudar de las decisiones adoptadas, sobre todo aquellas relativas a la lista de espera de trasplante. Un participante sintetiza la opinión de los demás cuando señala:

"Eso yo lo reconozco. Es una lista muy seria [la del INDT]. Este asunto de los donantes, nosotros sabemos... que no se podía saquear la lista, sabíamos. Teníamos que esperar con paciencia [por un órgano] y así fue”.

Otro aspecto que destacan es el trato de los profesionales de la salud, particularmente de médicos y enfermeras de los institutos o centros donde fueron trasplantados y les dan seguimiento. En este 
caso reconocen que no sólo establecen relaciones profesionales con dicho personal, sino que el trato llega a la amistad. Por ello, unánimemente consideran que reciben un buen trato, según lo señala una mujer participante:

"[Los médicos] son amigos. Yo llamo por teléfono [al hospital] y pregunto alguna cosa... y ellos me atienden, saben bien quién soy. Hace mucho ya [que me conocen]. Cuando pasé ese mes [internada] ellos estuvieron allí. Venía uno y otro... y todos con un [gran] cariño...”.

Los resultados exitosos de los trasplantes es otro de los reconocimientos hechos por quienes participan en este estudio. El mismo lo expresan en dos sentidos. Discrepando de la opinión de los profesionales de la salud cuando afirman que el mismo significa volver a la normalidad; para ellos el trasplante renal significa volver a nacer, tener otra oportunidad de vivir o una forma de salir de la hemodiálisis. Una mujer de mediana edad alude a la forma como lo entiende:

"Que maravilloso es tener un riñón nuevo. Yo digo que le cambia totalmente la vida a todo el mundo; es una cosa maravillosa... La sensación es que uno parece que nace de nuevo. A mí me dio la impresión de que nacía de nuevo, porque no tenía que ir más a diálisis...".

El trasplante también les significa la posibilidad de ser más independientes. Entre otras razones, porque les permite sentirse en libertad de llevar a cabo actividades imposibles de realizar durante la terapia de hemodiálisis, tales como comer, visitar amigos, trabajar y, consecuentemente, obtener sus ingresos propios.

\section{Teniendo derecho a la salud}

El segundo eje temático construido a partir de las narraciones de los participantes trata del derecho a la salud. La postura adoptada comúnmente por quienes se involucraron en este estudio es insistente en cuanto a que el acceso gratuito a una atención sanitaria oportuna y de calidad es un derecho humano. En consecuencia, la atención renal la entienden como un asunto al cual tienen derecho, más que visualizarlo como una dádiva o un favor. Los comentarios son de diversa índole, pero su tono es más o menos semejante al comentario de un participante cuando enfatiza la necesidad de exigir sus derechos:

"Todos tenemos nuestras obligaciones, pero tenemos nuestros derechos. Tenemos que exigir que cumplan nuestros derechos también. Porque si no [lo hacemos] siempre nos exigen: 'tiene que sacar', 'haga la cola', 'espere,' 'saque el número', 'espere 30 días para que lo vea el médico....”.

\section{Enfrentando problemas en la atención sanitaria}

El tercer eje temático apunta a la identificación de los obstáculos que enfrentan en la atención vinculada al trasplante renal. La impresión inicial que trasmiten los participantes es que tienen pocas dificultades, debido al acceso gratuito a la atención médica y particularmente a las terapias renales. Pero a medida que se avanza en las entrevistas va emergiendo una realidad cada vez más compleja. Algunos problemas afectan a todos los individuos enfermos, mientras que otros los viven algunos de ellos.

Los obstáculos en común. La Tabla 1 presenta los problemas que enfrentan los participantes de este estudio, los cuales se acompañan de sus citas respectivas. Según se puede observar, destacan temas variados y en niveles diferentes. Entre ellos, la falta de reconocimiento de los problemas sanitarios por parte de directivos y administradores, las contradicciones en el discurso oficial, la falta de acceso a la información, la peregrinación constante de los individuos enfermos y el maltrato de que son objeto en determinadas instituciones.

Los problemas específicos. A diferencia del rubro anterior, tres problemas afectan a sectores específicos de la población con trasplante renal. Uno de ellos es el centralismo de las instituciones involucradas en la atención renal, por el hecho de que los centros de trasplantes, el FNR y el INDT se ubican en Montevideo. Por tal motivo, quienes viven en el interior del país deben viajar con frecuencia a la capital por motivos ligados al tratamiento médico; entre ellos a las consultas de seguimiento, la hospitalización, recibir los medicamentos inmunosupresores y la realización de estudios clínicos. Un participante hace énfasis en el asunto en términos que no deja lugar a dudas:

"Tener que venir acá a [la calle] 18 [de Julio en Montevideo, al FNR] a buscar medicamentos todos los meses es un delirio. Ya es fastidioso por más que viva uno a 40 cuadras [del FNR], pueda ir en cualquier 


\section{Tabla 1}

Obstáculos que enfrentan los individuos con insuficiencia renal y trasplantados en la atención sanitaria.

Obstáculos Citas textuales

Falta de reconocimiento de problemas por parte de directivos

No cumplimiento de acciones reportadas Falta de acceso a la información Peregrinación constante

Maltrato a personas enfermas

Viviendo en la incertidumbre
“Vinieron del Ministerio de Salud Pública a presentar un plan de trabajo... Cuando hablaba esta gente daban a entender como si todo estuviera funcionando. Les digo 'ustedes están diciendo que no hay problema con la atención de los usuarios...' La gente tiene problemas. Se levanta a las 3 1⁄4 de la mañana y llega acá a las 6... ¿Y sabes qué pasa? El médico suspendió la consulta, no fue a trabajar. Nadie le avisa a la gente, nadie. Entonces ¿cómo me decís que esto está funcionando?"

"Si yo me quejo [del sistema sanitario] es de lo malo. Yo no puedo quejarme de que antes era peor y ahora está mejor. Se ha mejorado en muchas cosas, pero hay muchas cosas que se han difundido y no se cumplen"

"El Fondo Nacional de Recursos... no funciona realmente como debería funcionar. Tenemos el gravísimo problema de que no tenemos acceso [a la información]; es muy complicado tener acceso a la información. Yo digo que no es cristalina" "Voy al instituto de trasplantes cada tres meses, voy con mi nefrólogo cada tres meses, con el dermatólogo dos veces al año, con el oculista... con el dentista tengo que cuidar las infecciones. Me tengo que hacer ecografía de esto, de no sé cuánto... Tengo que ir al Fondo [Nacional de Recursos] todos los meses a buscar los medicamentos... a la Sociedad todos los meses a buscar otros medicamentos..."

"Los del Fondo [Nacional de Recursos] no son tan divinos... Vos vas ahí porque necesitás... Pero a veces te atienden tan mal que te hacen sentir como que fueras a pedir, como si fueras no sé qué. Todavía que tenés una enfermedad, te ponen una carguita más. Te tratan mal. ¿Qué necesidad?"

"El trasplantado renal tuvo la suerte de ser tocado por una varita para que le pusieran un riñoncito. Pero no tuvo la suerte de tener esa tranquilidad, de ese respaldo atrás. Cuando uno tiene dudas quiere consultar, [pero] no sabe a dónde tiene que ir"

Fuente: elaboración própria.

momento, tenga el horario y todo. Ahora, si vivo en Artigas o en Paysandú o en Tacuarembó ya es infinitamente rompedor de...".

Otro problema apunta a las dificultades que enfrentan quienes están afiliados al sector público, a diferencia de aquellos del sector privado. Quienes están en el sector público -específicamente adscritos a ASSE- refieren tres tipos de problemas: falta de medicamentos, largas filas de espera y la situación particular de quienes tienen menos recursos económicos. El primero no trata de carencias de los medicamentos inmunosupresores que entrega el FNR; el mismo se refiere a la falta frecuente de aquellos medicamentos que les fueron indicados para el tratamiento de sus diversas condiciones crónicas, pero que no encuentran siempre en los centros de salud. El segundo remite a los largos tiempos de espera para las consultas médicas, especialmente con los médicos especialistas. Una joven mujer que ha vivido con un trasplante renal durante años comenta del asunto:

"En Salud Pública está bravo con ese tema de darte día y hora [para las consultas]. Los médicos, igual te ponen urgente, pero no hay especialistas o está mucha gente anotada. Entonces... a veces tenés que esperar esperar y esperar".

La situación de aquellos enfermos renales que viven en condiciones precarias, o en la pobreza, es el tercer problema que aparece en las preocupaciones de quienes están afiliados a ASSE, sobre todo del interior del país. El asunto lo plantean en términos de que cualquier obstáculo adicional en la atención de este grupo tiene efectos directos en sus ya maltrechas condiciones. Una mujer de Rivera hace eco de lo dicho por outros:

"Tener que ir a Montevideo a buscar la medicación, perder de trabajar, si tenés plata y si no tenés. El hospital da un pasaje, pero hay gente que no se da cuenta, gente pobre, (...). A veces no tienen ni para comer, ni para el ómnibus, ni para nada...”.

La situación de quienes están afiliados al sector de las mutualistas, pareciera ser mucho mejor. Sin embargo, aquí se refieren dos problemas con insistencia: los gastos crecientes de bolsillo, particularmente en el caso de los jubilados, y el descuento en sus ingresos. Teniendo presente que tienen acceso gratuito a la atención sanitaria, este grupo externaliza una serie de preocupaciones por los gastos que 
hacen para seguir el tratamiento médico y evitar el rechazo del órgano trasplantado. Además de la cuota mensual suplementaria que pagan a la mutualista a la que están afiliados, refieren el pago de los ticketes. Las cifras son variables en las mutualistas, pero en una de ellas el costo de la consulta general era aproximadamente de 4 USD, la consulta de especialistas de 8 USD, los estudios clínicos básicos de 13 USD, los estudios especiales de 15 USD, además del ticket de cada medicamento prescrito. El asunto es particularmente preocupante para los adultos mayores, jubilados o pensionados y con varias enfermedades crónicas, ya que dichos gastos pueden ascender a $20 \%$ de sus ingresos. Uno de los participantes de Paysandú se refiere al tema:

"Cuando mi hija estaba en Montevideo me mandaba los medicamentos [cuando los recogía en el FNR]. Tengo mi cuñada [allá], pero no puedo estar molestándola todos los meses. A veces iba yo, y sacaba la cuenta entre lo que pagaba de pasajes, más medicamentos, más consulta, más la Sociedad. Digo 'esto no tiene gollete”.

Quienes están afiliados a las mutuales también mencionan el descuento de haberes de que son objeto, al dejar de laborar los días en que realizan alguna actividad relacionada con el seguimiento médico. Ello sucede cuando acuden a consulta, recogen medicamentos en el FNR o realizan algún trámite médico. Habla una joven con trasplante renal de Paysandú, que viaja con frecuencia a Montevideo por tales asuntos:

"Estás gastando de tu bolsillo... tenés que pagar la consulta... más el medicamento... ese te lo pagás aparte. Además ese día [de la consulta] me lo descuentan porque falto a trabajar. ¡Claro!, te descuentan el día... porque es una falta con aviso...".

\section{Formulando propuestas de acción}

El cuarto eje temático que emerge del análisis de la información trata de las propuestas y sugerencias de los participantes para mejorar el sistema de atención (renal). Según la mayoría, más que criticar al sistema de salud, les interesa hacer propuestas y sugerencias para resolver los problemas. En la Tabla 2 se presentan las principales propuestas, acompañadas de sus citas textuales. Entre ellas aparecen algunas tan generales como intentar la solución de los problemas mediante la negociación.

En cambio, hay otras específicas que responden a problemas concretos que viven en el día a día; destaca la de descentralizar los servicios de salud y la entrega de los medicamentos donde viven, sea en sus casas o en alguna instancia sanitaria del departamento. Como se desprende de la revisión de la tabla, tales propuestas difieren entre quienes viven en Montevideo respecto a los del interior; también entre quienes están afiliados al sector mutual frente a los del sector público. Entre otras, destaca la de dar prioridad a quienes habitan en el interior del país sobre los de Montevideo, no bajo el principio de igualdad, sino el de equidad.

\section{Discusión}

Este trabajo se propuso examinar la atención médica que se brinda a individuos con trasplante renal desde el punto de vista de quienes viven con el padecimiento, así como el de sus familias. Las contribuciones de un estudio de esta naturaleza radica en que presenta evidencias sobre la atención sanitaria que se otorga a quienes padecen insuficiencia renal en un país latinoamericano con un sistema integrado de salud; a la par que consolida una línea de trabajo interesada en recuperar la voz de diversos actores sociales para fortalecer su participación en el campo sanitario. Desde la perspectiva adoptada, la importancia de estudios de este tipo radica en que presta atención a los individuos enfermos y sus familias haciendo visible un saber, que si bien no es credencializado, se basa en la formación, la experiencia y la interacción social 24 .

Otra novedad de nuestros hallazgos radica en que los participantes enmarcan la atención médica vinculada al trasplante renal en el contexto sanitario del país y como un derecho a la salud. Los cuatro ejes sobre el cual gira su discurso reflejan una visión que va más allá del mundo subjetivo; ello en la medida que destacan elementos estructurales que permiten entenderla en el marco del sistema sanitario. Un dato al respecto es el reconocimiento que hacen de los avances del sistema de atención (renal), incluyendo a los organismos involucrados, antes de referirse a determinados obstáculos y carencias. Este reconocimiento coincide con hallazgos de otros estudios en los cuales se reporta que la población 
Tabla 2

Principales propuestas de los individuos con insuficiencia renal que han sido trasplantados para mejorar la atención sanitaria y sus citas correspondientes.

\section{Propuestas/Sugerencias}

Priorizar la negociación

Descentralizar los servicios sanitarios

Tratar a las personas enfermas de forma equitativa

Disminuir los tiempos de espera

Entregar los medicamentos en donde viven las personas enfermas

Hacer transparente la información

\section{Citas textuales}

“Cuando llamé al diario me dicen 'la muchacha que se dedica a salud no está, llámala'. La llamo, le conté la historia, me dice 'podes hacer una carta... y la publicamos... O si vos querés yo conozco un directivo de xxx y de repente puedo hacer una gestión'. Entonces vi que podía haber una solución sin tener que hacer más nada. Le digo 'bueno, dale...' Al rato me llamaron. Nunca más tuve problemas"

"Cuando esperamos la consulta hablamos... de descentralizar el lugar del control médico. La gente que vive en Artigas tiene que atravesar el país para [estar] 15 minutos como máximo con el médico; a no ser que sea una persona que tenga muchos problemas. Pero si no, no más de unos minutos..."

"Empezaría por eliminar la burocracia. Estudiar caso por caso, no hacer una ley general. Ahí se está teniendo a todo el mundo por igual. La justicia es tratar de forma desigual a quien es diferente. Porque si yo lo trato a usted de la misma forma que a aquel que no tiene nada... lo estoy favoreciendo a usted"

"Si fuera presidente lo que haría primero es un hospital o una sociedad en la que todos los que somos trasplantados, y en diálisis, estuviéramos atendidos en el mismo lugar... Me gustaría que todo estuviera en un hospital [o en la sociedad] y nos atendieran bien. Y que [la consulta] de los especialistas fuera más rápida, la atención también"

“Del Fondo [Nacional de Recursos]... Dios quiera que puedan traer los medicamentos a todos los Departamentos. Alcanzarlos [a las personas] para que sigan tomando su medicación como debe ser"

"La otra parte tiene que ser cristalina para poder [avanzar]... Tienen la obligación de que si se le pide determinada información, la tienen que dar"

Fuente: elaboración propia.

uruguaya valora favorablemente el sistema sanitario, particularmente a organismos como el FNR 25. Pero no puede pasarse por alto el hecho de que estos resultados discrepan de hallazgos reportados en otros estudios en los cuales los participantes suelen enfocarse en cuestiones específicas, tal sería el cumplimiento del tratamiento médico 26.

El sistema de atención (renal) uruguayo es superior a los de otros países de la región -Bolivia, Perú y Paraguay-, según los participantes de este estudio. Tal superioridad la conciben bajo el argumento de que la población de Uruguay tiene acceso gratuito a las terapias renales, mientras que sólo un porcentaje reducido de la población lo tiene en aquellos países. Más que verificar la precisión de las cifras, un señalamiento de esta naturaleza invita a explorar la percepción de la población con ERC sobre las terapias renales en tales países. En el mismo sentido, invita a realizar estudios comparativos en la región, entre ellos con aquellos países que brindan acceso gratuito y universal a las mismas terapias -Argentina y Brasil- y con otros vinculados al mercado -Chile y Colombia 27.

El reconocimiento de los logros del sistema de salud (renal) uruguayo no impide dejar de reconocer los obstáculos que enfrentan quienes han sido trasplantados. Dejando de lado los problemas que viven todos ellos, los datos expuestos parecieran indicar que hay dos grupos para quienes el sistema de atención renal tiene significados diferentes, y genera desigualdades en el acceso a la atención. Por un lado, para los jóvenes, trabajadores, de Montevideo y afiliados al sector de las mutualistas se trataría de un sistema accesible, eficiente y de calidad. En cambio, el mismo sería problemático para jubilados y pensionistas, del interior del país, con multi-morbilidad y pocos recursos económicos. Ciertamente, los problemas que enfrentan estos últimos son menores si se les compara con quienes viven en países de la región que carecen de un sistema de protección social en salud para toda la población 28, incluso para quienes cuentan con un sistema único de salud, como en lo relativo a los medicamentos en Brasil 29. Sin embargo, tal comparación no puede minimizar las dificultades que enfrentan los del segundo grupo. En el contexto uruguayo, tal situación podría cuestionar avances de la reforma sanitaria, y particularmente aquellas vinculadas a sus principios centrales, tales como los de justicia y equidad, sobre todo por la situación de desventaja de quienes viven en el interior y con menos recursos. 
Las propuestas de los participantes parecen lógicas, congruentes y factibles. Las mismas cuestionan aquella opinión de profesionales de la salud de que pacientes y familiares no se interesan por la enfermedad, ni el tratamiento. Al contrario, dejan al descubierto que a ningún participante se le había preguntado qué pensaba o proponía para mejorar su padecimiento renal o su manejo. Además, las mismas plantean un desafío a las instancias correspondientes, porque las obliga a darles algún tipo de respuesta. Un ejemplo sería el envío de medicamentos inmunosupresores a las casas de las personas enfermas o a las unidades hospitalarias ubicadas donde viven, en vez de seguirlos obligando a viajar a la sede del FNR en Montevideo. Estos asuntos invitan a preguntar si los organismos involucrados tienen conocimiento de tales propuestas, incluso si tienen la posibilidad de considerarlas seriamente, para apoyar la consolidación de la reforma sanitaria. Y no se trata de un dato menor, un asunto académico o que sólo competa al sistema sanitario uruguayo. También se trata de un asunto político que involucra en mayor o menor grado a los sistemas sanitarios de Latinoamérica, asunto a explorar en el futuro.

No queremos pasar por alto la ausencia de estudios en Uruguay, así como en la mayoría de los países latinoamericanos, sobre las percepciones, experiencias y prácticas de la población con ERC. El asunto, tema central de este trabajo, es pertinente por el creciente impacto que tiene esta enfermedad en países de la región, y porque se considera la participación social como pilar fundamental del sistema sanitario en varios países. Varias razones parecen explicar dicha ausencia. Al menos en el caso uruguayo, sostenemos que la misma se debe a la fuerte medicalización del sistema sanitario y de la misma sociedad uruguaya ${ }^{30}$. En tal contexto, la opinión de los individuos enfermos y sus familias no parece estar presente en la agenda de los profesionales de la salud, al considerarla no prioritaria para entender los daños a la salud o hacer propuestas de cambio al sistema de atención renal. Inclusive, pareciera que el tema pasa a segundo término al considerar que las terapias renales son un asunto menor, que afecta a un grupo minoritario de la población y se expresa en el ámbito hospitalario.

Dada la naturaleza del trabajo realizado, anotamos algunas limitaciones. Los resultados del estudio no son representativos de la población, ni generalizables al sistema sanitario o al país en su conjunto. Dadas las estrategias metodológicas empleadas, los hallazgos aplican a la población participante. Además, al haber centrado la atención en el punto de vista de quienes participaron, ello ha implicado dejar de lado sus significados, experiencias y prácticas, asuntos que habrá que explorar si queremos comprender la atención sanitaria tal como es vista, vivida, comprendida y enfrentada por la población. No olvidamos tampoco el haber dejado de lado al grupo mayoritario de enfermos renales, o sea, a aquellos en terapia dialítica y que podrían abundar sobre otros aspectos de la atención sanitaria en Uruguay, tal sería el caso de quienes son atendidos en el sector público y en el de las mutualistas.

No obstante lo anterior, el estudio tiene sus fortalezas. Los resultados podrían ser de utilidad para trasladarlos a determinadas circunstancias o utilizarlos como insumo para futuras investigaciones. Por ejemplo, para formular preguntas del tipo, ¿a cuánto ascienden los gastos de bolsillo entre quienes han sido trasplantados y tienen acceso gratuito a la atención sanitaria? Por otra parte, la información ha sido obtenida por el primer autor que ha trabajado con metodologías cualitativas durante décadas. Por ello nuestra certeza en la credibilidad de la información.

\section{Conclusiones}

Al reconocer el acceso gratuito y universal a las terapias renales, los individuos con trasplante de riñón valoran en términos positivos tanto la atención sanitaria, como determinados organismos involucrados. A pesar de ello, un sector importante -aquellos con bajos ingresos, del interior del país, adultos mayores, con multi-morbilidad, jubilados o pensionistas- parecen enfrentar obstáculos para acceder a una atención gratuita, oportuna y de calidad. Recomendamos impulsar estrategias para incorporar la voz de los individuos enfermos y sus familias, tanto para mejorar la atención renal, como para disminuir las desigualdades en el acceso a la misma. 


\section{Colaboradores}

F. J. Mercado-Martínez concibió el estudio, hizo la búsqueda bibliográfica, hizo el trabajo de campo, analizó la información y redactó el manuscrito. R. Levin-Echeverri concibió el trabajo, revisó la bibliografía, analizó la información y redactó el manuscrito.

\section{Agradecimientos}

A las personas que aceptaron participar en el estudio. Al personal de ATUR (Asociación de Trasplantados del Uruguay) por su valioso apoyo. A Daniela González, Mauricio Correa y Carlos Sánchez por las transcripciones. A Alejandra Toledo, Carlos Mera y los evaluadores anónimos por sus valiosos comentarios y sugerencias a una versión previa de este trabajo. Finalmente, al Consejo Nacional de Ciencia y Tecnología (CONACYT) de México por el apoyo brindado al primer autor (F.J.M.-M.).

\section{Referencias}

1. Perico N, Remuzzi G. Chronic kidney disease: a research and public health priority. Nephrol Dial Transplant 2012; 27 Suppl 3:iii19-26.

2. Port FK, Wolfe RA, Mauger EA, Berling DP, Jiang K. Comparison of survival probabilities for dialysis patients vs cadaveric renal transplant recipients. JAMA 1993; 270:1339-43.

3. Jha V, Garcia G, Iseki K, Li Z, Naicker S, Plattner B, et al. Chronic kidney disease: global dimension and perspectives. Lancet 2013; 382:260-72.

4. World Health Organization. Global status report on noncommunicable diseases 2010 . http://www.who.int/nmh/publications/ncd report_full_en.pdf (accedido el 09/Ago/2016).

5. De Vecchi AF, Dratwa M, Wiedemann ME. Healthcare systems and end-stage renal disease (ESRD) therapies. An international review: costs and reimbursement/funding of ESRD therapies. Nephrol Dial Transplant 1999; 14 Suppl 6:31-41.

6. Rucker D, Hemmelgarn BR, Lin M, Manns BJ, Klarenbach SW, Ayyalasomayajula B, et al. Quality of care and mortality are worse in chronic kidney disease patients living in remote areas. Kidney Int 2011; 79:210-7.

7. Tong A, Howell M, Wong G, Webster AC, Howard K, Craig JC. The perspectives of kidney transplant recipients on medicine taking: a systematic review of qualitative studies. Nephrol Dial Transplant 2011; 26:344-54.

8. Crowley-Matoka M. Desperately seeking "normal": the promise and perils of living with kidney transplantation. Soc Sci Med 2005; 61:821-31.

9. Denhaerynck K, Dobbels F, Cleemput I, Desmyttere A, Schäfer P, Schaub S, et al. Prevalence, consequences, and determinants of nonadherence in adult renal transplant patients: a literature review. Transpl Int 2005; 18 : 1121-33

10. Barendse SM, Speight J, Bradley C. The renal treatment satisfaction questionnaire (RTSQ): a measure of satisfaction with treatment for chronic kidney failure. Am J Kidney Dis 2005; 45:572-9.

11. Sullivan M. The new subjective medicine: taking the patient's point of view on health care and health. Soc Sci Med 2003; 56:1595-604.

12. Task Team for the Global Thematic Consultation on Health in the Post-2015 Development Agenda. Health in the post-2015 agenda. Report of the Global Thematic Consultation on Health. http://www.worldwewant2015.org/ file/337378/download/366802 (accedido el 09/Ago/2016). 
13. Ministerio de Salud Pública. Sistema de salud en Uruguay. In: Giovanella L, Feo O, Faria M, Tobar S, organizadores. Sistemas de salud en Suramérica: desafíos para la universalidad, la integralidad y la equidad. Río de Janeiro: Instituto Suramericano de Gobierno en Salud; 2012. p. 728-75.

14. Álvarez I, Bengochea M, Mizraji R, Toledo R, Saldías MC, Carretto E, et al. Three decades of the history of donation and transplantation in Uruguay. Transplant Proc 2009; 41:3495-9.

15. Ministerio de Desarrollo Social. Reporte Uruguay 2015. http://www.opp.gub.uy/images/ ReporteUruguay2015_OPP_web.pdf (accedido el 20/Feb/2016).

16. Freigedo M, Fuentes G, Araújo MR. Construyendo una coalición para romper el paisaje congelado: alcances y límites de la reforma de la salud en Uruguay (2005-2014). Íconos 2015; 19:49-66.

17. Olesker D. Igualdad, universalidad y accesibilidad: economía política y estado actual de la política pública de salud. In: Fernández Galeano M, Levcovitz E, Olesker D, coordinadores. Economía, política y economía política para el acceso y la cobertura universal en salud en Uruguay. Montevideo: Organización Panamericana de la Salud; 2015. p. 9-45.

18. Levcovitz E. La contribución del abordaje de economía política del sector salud a la orientación estratégica de la cooperación de la OPS/ OMS en Uruguay. In: Fernández Galeano M, Levcovitz E, Olesker D, coordinadores. Economía, política y economía política para el acceso y la cobertura universal en salud en Uruguay. Montevideo: Organización Panamericana de la Salud; 2015. p. m-u.

19. Fondo Nacional de Recursos. ¿Qué es el FNR? http:/www.fnr.gub.uy/que_es_fnr (accedido el 26/Jul/2016).

20. González-Martínez F, Orihuela S, Alvarez I, Dibello N, Curi L, Nin M, et al. Development of the national kidney transplantation program in Uruguay. Transplant Proc 2015; 47:2336-9.
21. Lock M, Scheper-Hughes N. A critical-interpretive approach in medical anthropology: rituals and routines of discipline and dissent. In: Sargent CF, Johnson TM, editors. Medical anthropology: contemporary theory and method. London/Westport: Praeger; 1996. p. 41-70.

22. Teddlie C, Yu F. Mixed methods sampling: a typology with examples. J Mixed Methods Res 2007; 1:77-100

23. Hsieh HF, Shannon SE. Three approaches to qualitative content analysis. Qual Health Res 2005; 15:1277-88.

24. Giddens A. Consecuencias de la modernidad. Barcelona: Alianza Editorial; 1994.

25. Fondo Nacional de Recursos. El valor de dejarse observar. Miradas externas sobre el FNR. http://www.fnr.gub.uy/sites/default/files/pu blicaciones/FNR_miradas_externas.pdf (accedido el 08/Mar/2016).

26. Cedillo-Galindo H, Gracida C. Barriers and strategies for taking medicines in adult patients with renal transplantation. Transplant Proc 2011; 43:3364-6.

27. Laurell AEC. Políticas de salud en pugna: aseguramiento frente a sistemas universales públicos. Rev Latinoam Enferm 2016; 24:e2668.

28. Ascencio-Mera CD, López-Arellano O. Peregrinando por un trasplante renal. La perspectiva de las personas con y sin seguridad social. In: Mercado-Martínez FJ, Hernández-Ibarra E, coordinadores. Crónica de la enfermedad renal. Voces que escuchan o viven el padecimiento. Guadalajara: Universidad de Guadalajara; 2016. p. 145-76.

29. Rosa MB, Reis AMM, Perini E. Drug shortage: a public health problem. Cad Saúde Pública 2016; 32:e00086916.

30. Portillo J, Rodríguez J, coordinadores. La medicalización de la sociedad. Montevideo: Nordan; 1993. 


\section{Abstract}

This study examines renal care from the perspective of kidney transplant patients with chronic kidney disease (CKD), as well as their families. A qualitative study was performed in three cities of Uruguay. Using theoretical sampling, we selected 25 persons with CKD that were living with kidney transplants, in addition to five family members. Semi-structured interviews were held to explore the participants' perspectives, as well as content analysis. Four thematic lines were constructed from the participants' discourse: recognizing the successes of the system of care, the right to health, identifying problems in care, and formulating proposals for action. The system of care is rated as adequate, efficient, and high-quality by individuals living with kidney transplants who are young, employed, residing in the national capital of Montevideo, and covered by the private health sector. The opposite is true for retirees and pensioners, those with multiple morbidities, from inland Uruguay, covered by the public sector, and living in poverty. The participants also formulated proposals to solve the problems that were identified. In addition to acknowledging free and universal access to renal therapies to which patients feel they have the right, we conclude that kidney transplant patients assess the healthcare favorably and the agencies involved. Nevertheless, there are serious obstacles to accessing free, timely, and high-quality care. We recommend promoting strategies to incorporate proposals by patients and their families in order to improve such care and decrease social inequalities.

Chronic Renal Insufficiency; Kidney Transplantation; Health Systems

\section{Resumo}

Este trabalho examina a atenção renal desde a perspectiva daqueles que foram transplantados, sofrendo insuficiência renal crônica (IRC), assim como desde a das suas famílias. Foi realizado um estudo qualitativo em três cidades do Uruguai. Utilizando uma amostragem teórica, selecionamos 25 pessoas com IRC que viveram com transplante renal, e cinco familiares. Foram realizadas entrevistas semiestruturadas para explorar as perspectivas dos participantes; assim como a análise de conteúdo. Quatro eixos temáticos foram construídos a partir da intervenção dos participantes: reconhecendo os resultados alcançados com o sistema de atenção, tendo direito à saúde, identificando problemas na assistência e formulando propostas de ação. O sistema de assistência é considerado adequado, eficiente e de qualidade por quem vive com transplante renal, são jovens, trabalhadores, de Montevidéu e estão cobertos pelo seguro de saúde privado. O oposto acontece com os aposentados ou pensionários, com multi-morbilidade, no interior do país, cobertos pelo sistema público e vivendo na pobreza. Os participantes também apresentam propostas para resolver os problemas identificados. Além disso, se reconhece o acesso gratuito $e$ universal às terapias renais, para as quais consideram ter direito, concluimos que os indivíduos com transplante de rins valorizam favoravelmente a atenção sanitária e as instituições envolvidas. Porém, alguns enfrentam sérios obstáculos para aceder a uma atenção gratuita, adequada e de qualidade. Recomendamos incentivar estratégias para incorporar propostas dos indivíduos doentes e suas famílias, ao fim de melhorar a referida atenção e reduzir as desigualdades sociais.

Insuficiência Renal Crônica; Transplante de Rim; Sistemas de Saúde
Recibido el 15/Sep/2016

Versión final presentada el 03/Dec/2016

Aprobado el 01/Feb/2017 